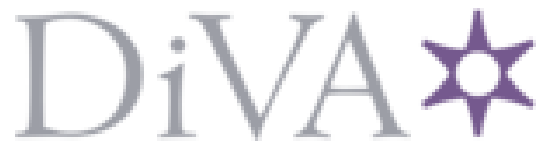

http://www.diva-portal.org

Postprint

This is the accepted version of a paper presented at IEEE 16th International Conference on Automation Science and Engineering (CASE).

Citation for the original published paper:

Tajvar, P., Barbosa, F S., Tumova, J. (2020)

Safe Motion Planning for an Uncertain Non-Holonomic System with Temporal Logic

Specification

In:

https://doi.org/10.1109/CASE48305.2020.9216891

N.B. When citing this work, cite the original published paper.

Permanent link to this version:

http://urn.kb.se/resolve?urn=urn:nbn:se:kth:diva-298449 


\title{
Safe Motion Planning for an Uncertain Non-Holonomic System with Temporal Logic Specification
}

\author{
Pouria Tajvar, Fernando S. Barbosa, Jana Tumova
}

\begin{abstract}
We propose a sampling-based motion planning algorithm for systems with complex dynamics and temporal logic specifications allowing to tackle sophisticated missions. By complex dynamics we refer to non-holonomy and disturbance that prevent implementation of an exact steer function. We instead construct a set of feedback motion primitives guaranteeing bounded state uncertainty (and thus safety) allowing the system to follow an arbitrarily long trajectory without replanning. The motion primitives allow to use $A^{\star}$-based algorithm to provably accomplish the temporal logic mission. We propose a heuristics for the $A^{\star}$-based algorithm via construction of backward trees. We illustrate the approach on several case studies, including simulations of a rover and fixed wing drone. We further show that construction of backward trees allows for faster re-planning compared to the state-of-the-art.
\end{abstract}

\section{INTRODUCTION}

Linear temporal logic (LTL) enables to conveniently express specifications in a high level manner, beyond what can be expressed as a sequence of reachability sub-tasks. Examples include periodic surveillance (repeatedly visit regions $\mathrm{A}$ and $\mathrm{B}$ ), sequencing (visit region $\mathrm{A}$ then $\mathrm{B}$ then $\mathrm{C}$ ), request-response (whenever you visit region $\mathrm{A}$, make sure to visit also region B after), and their combination. An LTL formula can be translated into an automaton that is suitable for automated analysis such as model checking. Algorithms for autonomous planning for satisfying LTL specifications were originally developed for systems with purely discrete states [1]. Recently, methods have been proposed to handle systems with continuous dynamics, such as robots.

Early approaches in temporal logic planning for robots are based on state abstraction, i.e. discrete representation through decomposition of the continuous state space into a countable set of sub-spaces [2], [3], [4]. The main drawback of state abstraction is that the system's discrete representation grows prohibitively large with the number of state space dimensions and also in problems where a fine granularity of discretization is required. This is after all a drawback of all abstraction based planning approaches, not only the ones dealing with temporal logic specifications.

Sampling based motion planners avoid the explicit states space construction and thus mitigate the dimensionality problem. Motivated by the success of algorithms, such as RRT [5] and its asymptotically optimal version $\mathrm{RRT}^{\star}$ [6], several approaches have been proposed in order to enhance

${ }^{*}$ Division of Robotics, Perception and Learning, KTH Royal Institute of Technology Stockholm, Sweden tajvar@kth. se, fdsb@kth. se tumova@kth. se. This work was supported by the EU H2020 Research and Innovation Programme under GANo. 731869 (Co4Robots) and the Swedish Research Council (VR). sampling-based algorithms with temporal logic mission specifications [7], [8], [9], [10].

However, application of sampling-based planning for temporal logics tasks is not trivial since the algorithm should consider robot's state-space and the task space (i.e. the automaton state) simultaneously. Hence, one of the main challenges in temporal planning as compared to reach-avoid planning is the size of the search space.

Various approaches have been proposed to address this issue, mainly through heuristics for biasing of the sampling towards satisfaction of the specification [11], [8]. The biasing approaches can be classified as geometry guided and automaton guided. One of the early works in this direction combines geometry and automaton guidance [11], [7] that is implemented as part of Open Motion Planning Library (OMPL) [12]. It proposes a two-layer planning scheme with a high-level planning layer over a discretization of the state space and a low-level planner that is sampling based. A recent work on multi-agent path planning from temporal logic specifications using $\mathrm{RRT}^{\star}$ uses visibility graphs as a geometrical bias for sampling [8]. While geometrical guiding is shown to significantly speed-up the search algorithm, it is restricted to two and in some cases small three dimensional environments. To address this limitation, we propose a guidance heuristic that does not rely on geometrical decomposition of the environment.

There has also been research towards integrating sampling based motion planning with feedback control laws to allow for systems with complex dynamics. For instance, LQR-trees [13] iteratively build stabilized sets on the state-space of the system. FaSTrack [14], on the other hand, uses an offline step to create a safe envelope around the planned trajectory to ensure collision avoidance. Such methods however, are not directly applicable to sampling based approaches that are designed for temporal logic specifications.

In terms of control synthesis, our approach is based on construction of robust motion primitives, where a multi-step feedback controller is designed for each motion primitive to ensure bounded uncertainty. A similar approach has been adopted in works on bounded and shrinking reachable sets such as funnel libraries [15] and contraction theory based control [16]. However, having a finite set of actions has the drawback of exponential search space growth with length of the trajectory which in turn increases with the complexity of the environment and the specification. As a result keeping the branching factor of the search algorithm low can significantly improve the scalability of such algorithms. Therefore our approach attempts to combine the scalability of sampling 
based motion planning with the safety guarantees provided by primitive based control synthesis for non-holonomic systems with disturbance via tree guided heuristics.

The contribution of this work concerns two of the challenges in motion planning from temporal logical specifications. The first contribution is the extension of LTL planning algorithm to systems with complex dynamics, integrating the motion planning carefully with feedback control laws. By complex dynamics we particularly refer to non-holonomy and disturbance that prevent implementation of an exact steer function, i.e. a control policy, that can drive the system from any initial state to an arbitrary target state. The second contribution is to provide a scalable planning algorithm that serve as heuristics in guiding the control synthesis.

\section{Problem Formulation}

\section{A. System}

Consider a robot with uncertain non-holonomic dynamics, modeled as a dynamical system

$$
\dot{x}=f(x, u)+w
$$

with $x \in X \subset \mathbb{R}^{n_{x}}$ and $u \in U \subset \mathbb{R}^{n_{u}}$ being the robot's state and inputs respectively, and $w \in W \subset \mathbb{R}^{n_{x}}$ being disturbance with known bounds. The dynamic function of the robot $f$ may be unknown but it can be sampled from.

A subset of the state space is occupied by some obstacles, denoted as $O \subset X$, and the free space, i.e. the complementary set of the obstacles, is denoted as $X_{\text {free }}=X \backslash O$.

A valid timed trajectory for the robot can be represented as a tuple $(\mathbf{x}, \mathbf{u}, \mathbf{w})$ where $\mathbf{x}: \mathbb{R} \rightarrow X, \mathbf{u}: \mathbb{R} \rightarrow U$, $\mathbf{w}: \mathbb{R} \rightarrow W$ and $\forall t \in \mathbb{R},\left.\frac{d x}{d t}\right|_{t}=f(\mathbf{x}(t), \mathbf{u}(t))+\mathbf{w}(t)$.

Let $R=\bigcup_{i=1}^{n_{r}}\left\{r_{i}\right\}$ be a set of the regions of interest in the state space $\left(r_{i} \subset X\right)$, for example load/unload areas, charging station etc. We assume that the regions of interest are disjoint. We define the trace $\boldsymbol{\sigma}(\mathbf{x})$ of a trajectory $\mathbf{x}$ as the sequence of regions visited by the robot along the trajectory. For example $\sigma(\mathbf{x})=" /, \mathbf{r}_{\mathbf{1}}, /, \mathbf{r}_{\mathbf{2}} "$ means that, along $\mathbf{x}$, the robot starts outside any region of interest, enters $r_{1}$, exits $r_{1}$ and enters $r_{2}$.

\section{B. Mission}

A mission for the robot is defined as a linear temporal logic (LTL) formula $\Phi$ over the regions of interest. LTL is recursively built according to the following grammar.

$$
\phi:=r|\neg \phi| \phi_{1} \wedge \phi_{2}\left|\phi_{1} U \phi_{2}\right| \bigcirc \phi,
$$

where $r \in R, \neg$ and $\wedge$ are the standard Boolean operators, $\bigcirc$ is a temporal operator requiring $\phi$ to hold next, and $U$ is a temporal operator requiring $\phi_{1}$ to hold until $\phi_{2}$ happens. Furthermore, two useful operators are derived: $\diamond \phi=$ true $U \phi$ and $\square \phi=\neg \diamond \neg \phi$ represent $\phi$ holding eventually and always. For instance, $\diamond r_{1} \wedge \diamond r_{2}$ requires $r_{1}$ and $r_{2}$ to be eventually visitied (in an arbitrary order) while $\square \neg r_{3}$ then says that $r_{3}$ should be always avoided.

LTL formulas can be translated to Büchi automata. We will denote such translation of $\phi$ as the tuple $B_{\phi}=$ $\left(Q_{B}, \Sigma_{B}, \Delta_{B}, I_{B}, F_{B}\right)$, where $Q_{B}$ is the set of automaton

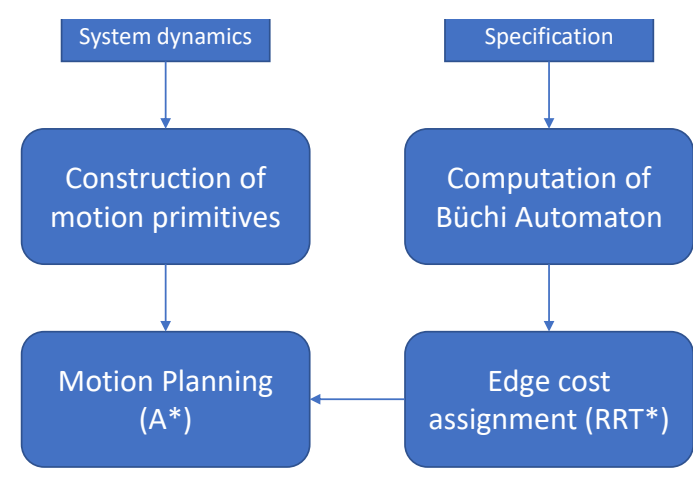

Fig. 1: Approach overview

states, $\Sigma_{B}=R \cup\{/\}$ is the alphabet, $\Delta_{B}: Q_{B} \times \Sigma_{B} \rightarrow Q_{B}$ is the transition function and $I_{B}$ and $F_{B}$ are the initial and the final states respectively. A word is accepted by $B_{\phi}$ if a state in $F_{B}$ is visited infinitely often; the set of all words is a language, denoted by $L(B)$.

A control policy $\pi:\left(B_{\Phi}, X\right) \rightarrow U$ is a mapping between the augmented state of the robot and the input space.

\section{Problem statement}

Problem 1: Given a robot with uncertain non-holonomic dynamics (1), a set of obstacles $O$, regions of interest $R$, and an LTL mission specification $\Phi$ over $R$, find a control policy $\pi$ such that all valid timed trajectories $(\mathbf{x}, \pi(\mathbf{x}), \mathbf{w})$ are collision free and produce traces that satisfy $\Phi$.

\section{METHOD}

An overview of our approach is shown in Fig. 1. To address Problem 1, given that we may not have explicit access to the dynamic function $f$, our approach is based on datadriven construction of robust motion primitives explained in III-A. We can then search for a trajectory that satisfies the specification as a sequence of motion primitives. To guide our search for a satisfying sequence, we have proposed a heuristic algorithm, explained in section III-B. III-C.

\section{A. Construction of motion primitives}

The motion primitives for the mobile robot are constructed as proposed in our previous work [17]. The method is based on data-driven computation of local feedback control laws for each motion primitive and exploits the translation invariance property of the dynamics of a mobile robot.

The input-space $U$ of the robot is divided into sub-regions $\hat{u}$, where $\hat{u}$ is represented by a central value $\hat{u}_{0} \in U$ and size vector $\sigma_{\hat{u}}$ of a rectangular neighborhood, giving: $\hat{u}=$ $\left\{\hat{u}_{0}\right\} \oplus\left[-\sigma_{u}, \sigma_{u}\right] \subset U$.

In each region, an affine discrete-time model of the dynamics $\hat{f}$ and a disturbance bound $\hat{\mathbf{W}}$ is derived by collecting data samples corresponding to that region such that

$$
\forall u \in \hat{u}, \bar{f}(x, u)=\{A x+B u+c\} \oplus \mathbf{W} .
$$

The model parameters can be found as the solution to a linear programming problem that minimizes $\mathbf{W}$ bounds with (2) as constraints for the data samples. 
A motion primitive $C_{\hat{u}}\left(x, x_{r e f}\right)=\hat{u}+\hat{B} \times\left(x_{r e f}-x\right)$ is then constructed for the region $\hat{u}$ as a multi-time-step feedback control law. Multi-time-step feedback means that the corrective action for state error in one step spans over multiple time steps. This is particularly essential for nonholonomic systems where the reachable set is degenerate and the error in an arbitrary direction cannot be compensated in a single time step.

It is shown in the proof of Theorem 1 in [17], that a multitime-step feedback control law can be found if the system is $n$-step robustifiable, which is the discrete-time equivalent of being small time locally attainable (STLA).

The implication of this result is that we can compute a bounded reachable set for execution of each motion primitive, and as we chain the motion primitives, the size of the reachable set remains within the same bounds allowing us to verify a collision free trajectory before execution.

\section{B. Extended Büchi automata}

We first translate the LTL specification $\Phi$ to a Büchi automaton $B=\left(Q_{B}, \Sigma_{B}, \Delta_{B}, I_{B}, F_{B}\right)$ using the LTL2BA algorithm [18]. We then use $B$ to derive an extended automaton $B^{\dagger}=\left(Q_{B^{\dagger}}, \Sigma_{B^{\dagger}}, \Delta_{B^{\dagger}}, I_{B^{\dagger}}, F_{B^{\dagger}}\right)$ where each state $q^{\dagger} \in Q_{B^{\dagger}}$ is a 2-tuple $(q, \sigma)$ of a state $q \in B$ and a letter (guard) $\sigma \in \Sigma_{B}$ that has led to that state; therefore, each state in $B^{\dagger}$ can be reached by exactly one letter, i.e. reaching one region of interest. The derivation of $B^{\dagger}$ is detailed in Alg. 1.

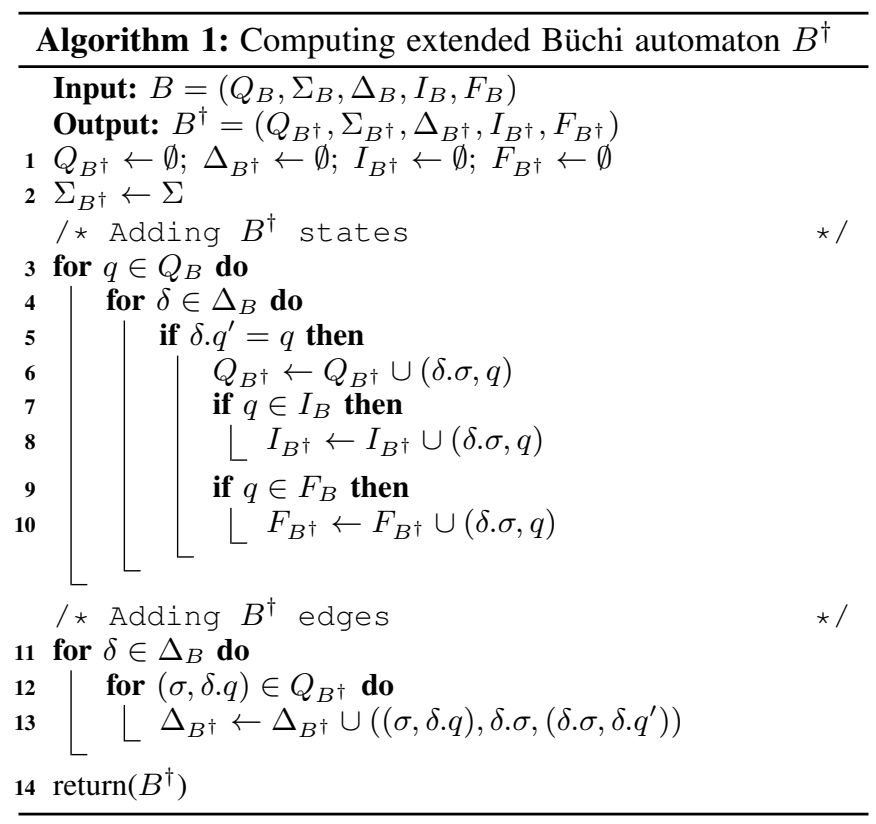

Lemma 1: $L(B)=L\left(B^{\dagger}\right)$.

Proof: Since bisimulation implies language equivalence [19], it is sufficient to show that $B^{\dagger}$ bisimulate $B$ :

Let the equivalence relation $\mathcal{R}$ be: $\mathcal{R}: \bigcup\{((\sigma, q), q)\}$. It can be readily seen that a word $\delta=\left(q, \sigma^{\prime}, q^{\prime}\right)$ is enabled in both automatons and results in $\left(\sigma^{\prime}, q^{\prime}\right)$ and $q^{\prime}$ in $B^{\dagger}$ and $B$ respectively. By definition we have $((\sigma, q), q) \in \mathcal{R}$.

Intuitively, $B^{\dagger}$ holds track of the last word and other than that, follows the same dynamic as $B$.

\section{Construction of the heuristic trees}

In order to estimate the distance of a certain state from satisfying the specification, we will provide a cost-to-go measure to each region of interest from any point in the state space. To do so, we use RRT ${ }^{\star}$ to construct a backward tree for each $r_{i} \in R$. The tree construction algorithm can be followed in Alg. 2. The tree $\tau_{i}$ is constructed backwards as the root of the tree is initialized in $r_{i}$ [line 2]. A node $\eta$ in the tree is represented with a 3-tuple $\eta=\left(p_{\eta}, \mathbf{x}_{\eta}, c_{\eta}\right)$ with $p_{\eta}$ corresponding to $\eta$ 's parent node and $\mathbf{x}_{\eta}$ corresponding to the trajectory connecting $\eta$ 's state $x_{\eta}$ to it's parent node; $c_{\eta}$ is the cost of going from $x_{\eta}$ to the tree root [line 3]. In each iteration, a random point $s$ is sampled in the state space, $s$ is then steered to the closest node $n$ in the tree [lines 4-6].

Recalling that the tree is only used to provide a heuristic for the main control synthesis, we can use any simplified dynamics that permits finding an exact steer function.

Similar to forward trees, using a backward tree constructed with $\mathrm{RRT}^{\star}$ results in a probabilisticaly complete and asymptotically optimal algorithm. The benefit of having a backward tree for a region of interest is that the tree is reusable from different initial states encoding an estimate of the cost-to-go to the corresponding region.

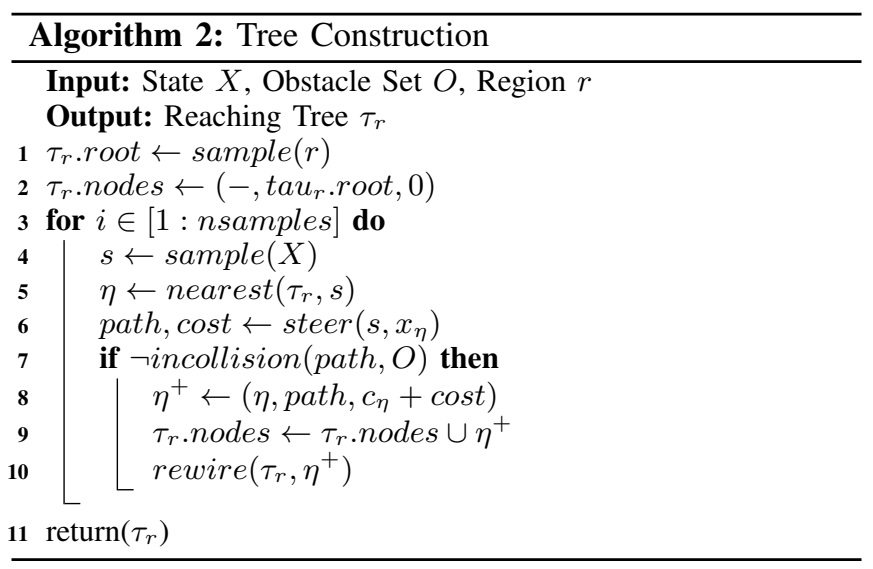

1) Computation of the heuristic for a state: Having computed a backward reaching tree for each region of interest, we can now construct a heuristic function that estimates the distance from an extended state $(b, x)$ to the closest accepting state. The approach is summarized in Alg. 3. We introduce $G$ as a weighted replication of $B^{\dagger}$, where each edge $\delta$ has an additional weight attribute, i.e. the cost of going through that edge. To assign weight to each edge $\delta$, we use the tree that corresponds to the destination region $r$ that enables that transition.

If the origin of the edge $\delta$ coincides with the robot's Büchi state $b$, the edge weight is calculated by steering the robot's physical state $x$ to the nearest node in $\tau_{r}$ [lines 4 6]. Otherwise, the edge weight is calculated by steering the root of the tree corresponding to the edge's originating state region $r_{o}$ to the nearest node in $\tau_{r}$ [lines 8-10]. In any case, adding the steering cost to the nearest node $\eta$ 's distance to the tree root yields the edge weight. 


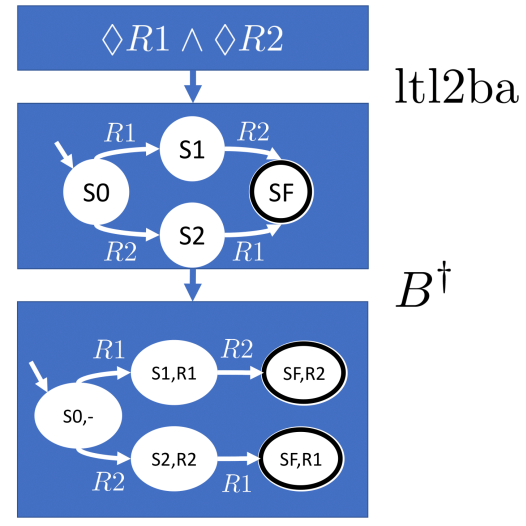

(a) Specification as Büchi automaton

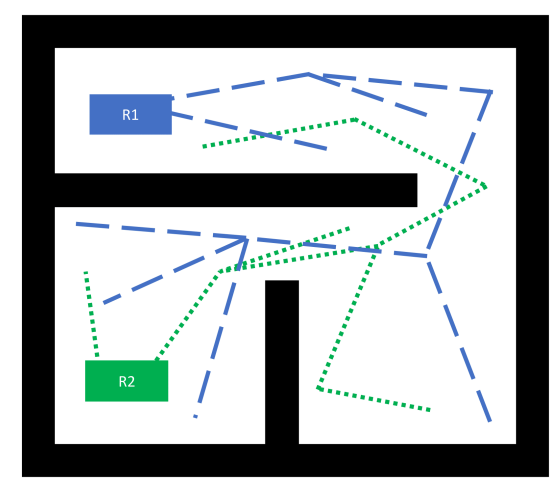

(b) Backward tree construction

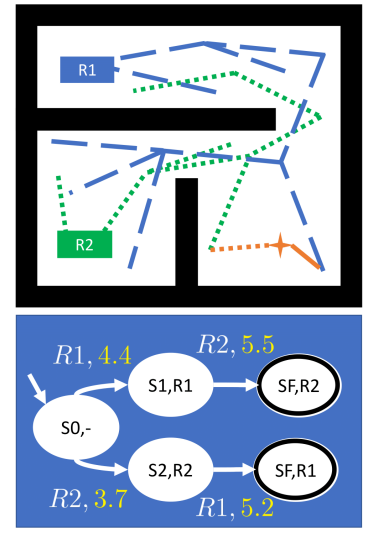

(c) Weight assignment

Fig. 2: Heuristic construction for evaluating a state: outgoing edges of the current Büchi state are weighted based on robot's current position and other edges use the default weights i.e. based on the distance of a region's root from it's target region.

With all of the edge weights computed, we can now find a satisfying solution that minimizes the cost through graph search and return the corresponding cost as the heuristic value. While all of edges are evaluated in Alg. III-C for simplicity of expression, it is evident that it is enough to calculate the costs of edges that do not originate from the state robot's current Büchi state $b$ once and only re-evaluate the edges that originate from $b$.

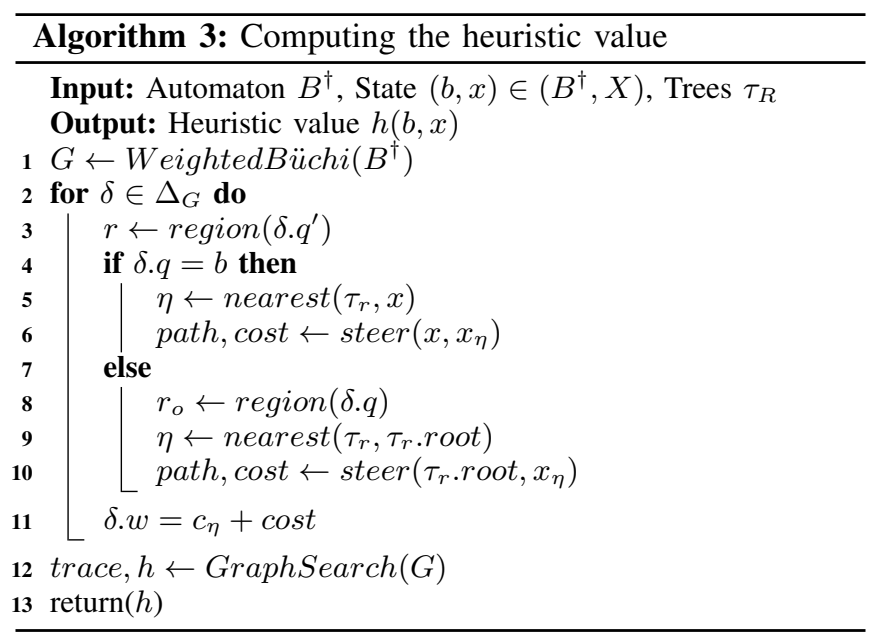

Remark 1: For a deterministic system where it is possible to implement a steer function. A satisfying solution to Problem 1 can be directly obtained from Alg. 3 since the trace output from graph search can be reproduced by following the backwards trees knowing that the root in each tree in the sequence can be steered to a node in the subsequent tree.

\section{Planning}

We now have a finite set of actions, i.e. the robust motion primitives, that due to the uncertainty preserving property, can be chained to synthesize an arbitrarily long sequence. We translate the problem into a planning problem on a discrete graph, where vertices represent centers of $\epsilon$-balls that do not intersect with the under-approximation of the collision space, and edges are defined by motion primitives driving the system from one center of an $\epsilon$-ball to another. The feedback control term ensures that regardless of where within the former $\epsilon$-ball the system is, it will end up within the latter $\epsilon$-ball. We use the Anytime Repairing $A^{\star}\left(A R A^{\star}\right.$ [20]) algorithm with the heuristic derived in section III-C. $A R A^{\star}$ is an implementation of $A^{\star}$ that allows pre-mature termination with shrinking bound on sub-optimality of the solution.

\section{CASE STUdies AND DISCUSSION}

We have run three main case studies. The first is on the efficiency of the heuristic construction with basic RRT ${ }^{\star}$ without any dynamics. The second is a rover with forward only motion primitives that follow Dubins model $\dot{x}=$ $\cos (\theta), \dot{y}=\sin (\theta), \dot{\theta}=u_{1}$. The last case study is a fixed wing model with planar dynamics similar to the Dubins model and bounded ascent/descent rate $\dot{z}=u_{2}$.

They are deployed in the environments illustrated in Figs. 3 and 4, with the red boxes illustrating the regions of interest $r_{1}, \ldots, r_{7}$, and $r_{1}, \ldots, r_{3}$, respectively. The missions are represented by LTL formulas $\bigwedge_{i \in\{1, \ldots, 7\}} \diamond r_{i}$ and $\bigwedge_{i \in\{1, \ldots, 3\}} \nabla r_{i}$ requiring an eventual visit to each region of interest.

The algorithm was implemented in Python 3.7 and the reported times were obtained on a standard laptop with 16GB memory and $2.8 \mathrm{Ghz}$ processor.

\section{A. Geometric biasing and re-usability}

As discussed in Rem. 1, the heuristic computation algorithm already provides a solution to the LTL motion planning problem to a deterministic system with the dynamics used for the building the trees. We therefore compare the time efficiency of this solution of building backward trees without dynamical constraints to [8], one of the fastest LTL motion planner for 2D systems without dynamics so far. We run each algorithm until a first solution is found.

[8] uses visibility graphs as a sampling bias for tree construction. Visibility graphs are effective tools for motion planning in $2 \mathrm{D}$ environments as they provide the shortest 


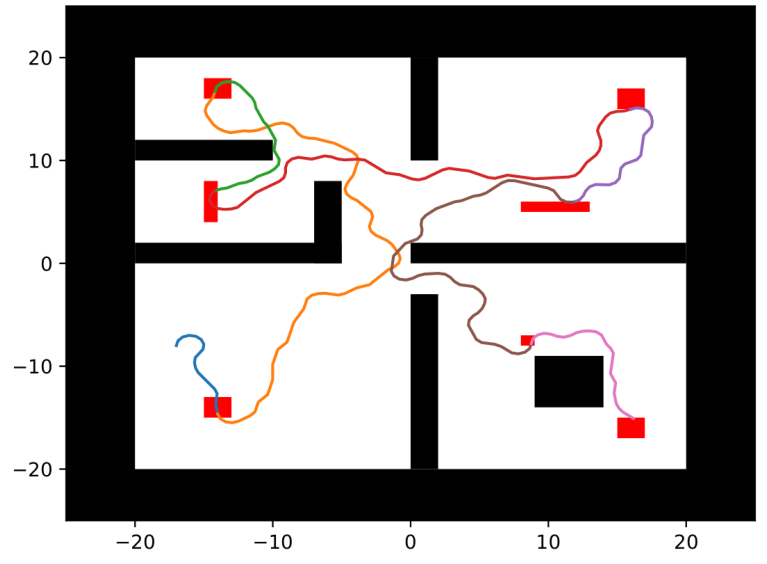

Fig. 3: Rover model with three motion primitives guided with tree without dynamics. Colors represent state of the automaton.

\begin{tabular}{|ccc|}
\hline Method & Geometric Biasing & Process time \\
\hline$[8]$ & Visibility graph & $1.3 \mathrm{~s}$ \\
\hline Backward tree & None & $12 \mathrm{~s}(80 \mathrm{~ms})$ \\
\hline Backward tree & Visibility graph & $1.5 \mathrm{~s}(-)$ \\
\hline
\end{tabular}

TABLE I: Planning without dynamics - 7 regions path to the goal; part of this path may of course be adjacent to the obstacles, nevertheless, it is informative about the direction of progress toward the goal. As can be seen in Table I, [8] is faster than the first invocation of the presented method by almost a degree of magnitude. By first invocation we mean when the backward trees are constructed. The same improvement can be seen in the presented method when the sampling is guided by the visibility graph. However, the main advantage of using backward trees is their re-usability with respect to position change, specification change and requestresponse tasks. As long as the environments and the region of interest remain unchanged, the same backward trees can be used to generate a new plan, these subsequent calls can be orders of magnitude faster (in this case $80 \mathrm{~ms}$ ). When using visibility graph, this advantage was lost since due to the sparsity of the tree, the robot at a different state would not be able to steer to the tree.

Furthermore, use of geometrical biasing such as visibility graph or triangulation as used in [7], limits the applicability of the motion planner to $2 \mathrm{D}$ environments. In the following section we will look into application of backward tree heuristics in dynamical motion planning and planning in a 3D environment.

\section{B. Dynamics and Higher-dimension problems}

Let us look at Fig. 3 again. We want to address the same motion planning problem of eventually visiting all of the regions. This time, the robot is subject to kinodynamic constraints. We have constructed three motion primitives for the robot. While in the previous problem without dynamics, it was possible to find a solution by having fewer than 200 nodes per tree, such sparse trees could not provide good heuristic measures and sometimes resulted in the ARA* algorithm getting stuck in local minima. Trees with 1000 nodes, however, provided informative heuristics and a solution was found within $12 \mathrm{~s}$ through the $\mathrm{ARA}^{\star}$.

We then applied the method to a simulated fixed wing drone model. The heuristic is again computed via construction of backward trees without dynamics. The computed trajectory is depicted in Fig. 4. It can be seen that even though the drone dynamics highly restricts it's motion, as for example it has to spiral to change altitude, the planner has been able to find a satisfying trajectory and the heuristic has significantly limited the search branching factor. For plans of length 28,75 and 31 , the $A R A^{\star}$ has run 61,171 and 51 iterations respectively. For an ideal heuristic, i.e. returning the exact distance to the goal, the number of iterations would be equal to the length of the plan. On the other hand, without using a heuristic, e.g. using Dijkstra, the number of iterations grows exponentially with the length of the plan, i.e. $9^{l}$ in case of the drone model. Therefore, given the relatively close number of iterations and path length suggests that tree driven heuristic is informative even without reflecting the dynamics.

\section{Computational complexity and run-time}

In terms of computational complexity, we can divide the proposed approach into three main sections: construction of backward trees, discrete LTL planning on the weighted Büchi automaton and dynamical planning with motion primitives. We have reported the run-time break down of each section in Table. II the different versions of the problem.

The backward reachable trees are constructed with 1000 nodes in all cases. As expected, computation time scales linearly with the number of regions of interest as the trees are constructed independently; it is however slightly lower for the 3D case as 1000 samples are more sparsely distributed in the 3D environment resulting in fewer rewirings.

Solving the discrete planning problem has taken a relatively small part of the process time in our problem set which, in terms of LTL formula size, is similar to the state of the art [21], [8], [2]. However, it appears to scale with a significantly higher order compared to tree construction due to the combinatorial nature of the problem. The worst case complexity of LTL is shown to be 2EXP-complete [1]; however, there are various fractions of LTL for which algorithms with lower complexity have been established [22], [23]. The presented method can benefit from approaches in formal synthesis to improve scalability with respect to the size of the formula.

Construction of motion primitives also scales linearly with the size of the formula, or more precisely the number of edges of the Büchi automaton that are in the discrete planning solution. However, the problem scales typically with a higher order with respect to the trajectory length depending on the heuristic. The worst case order of planning with motion primitives is exponential as discussed before, but the backward tree heuristic is shown to significantly improve the performance. 


\begin{tabular}{|cccc|}
\hline Problem & Trees & LTL planning & MP planning \\
\hline $2 \mathrm{D}-3$ & $12 \mathrm{~s}$ & $50 \mathrm{~ms}$ & $12 \mathrm{~s}$ \\
\hline $2 \mathrm{D}-7$ & $34 \mathrm{~s}$ & $70 \mathrm{~ms}$ & $33 \mathrm{~s}$ \\
\hline $2 \mathrm{D}-14$ & $61 \mathrm{~s}$ & $8 \mathrm{~s}$ & $80 \mathrm{~s}$ \\
\hline 3D-3 & $8 \mathrm{~s}$ & $22 \mathrm{~ms}$ & $44 \mathrm{~s}$ \\
\hline 3D-7 & $18 \mathrm{~s}$ & $40 \mathrm{~ms}$ & $69 \mathrm{~s}$ \\
\hline 3D-14 & $43 \mathrm{~s}$ & $23 \mathrm{~s}$ & $206 \mathrm{~s}$ \\
\hline
\end{tabular}

TABLE II: Run-time break down of the algorithm

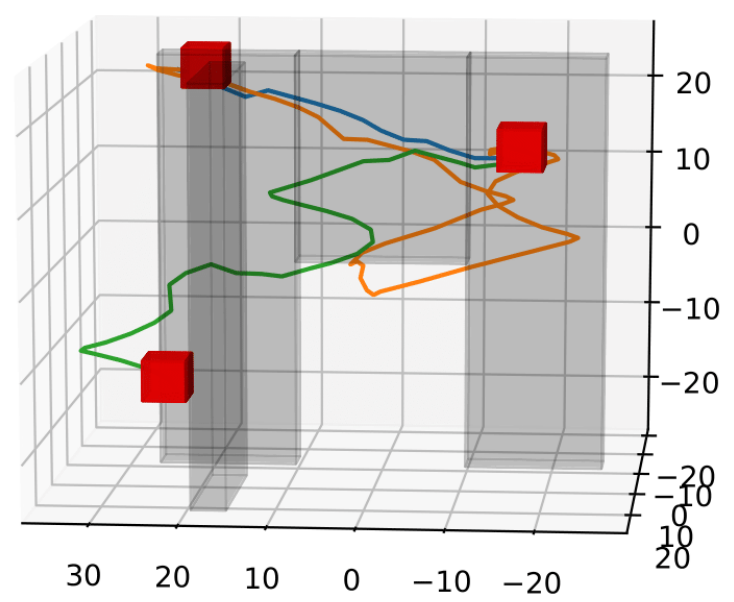

Fig. 4: Fixed wing model 9 motion primitives guided with tree without dynamics. The robot motion is restricted by descent and turn rate bounds.

\section{CONCLUSIONS AND FUTURE WORK}

We developed a motion planning algorithm from linear temporal logic specifications for non-holonomic robots subject to disturbance. We showed that backward reachable trees provide rich heuristics to guide the planning; they do not rely on geometric decomposition of the environment and they are re-usable for different specifications and robot states. The presented method relies on prior knowledge of the environment and unrestricted sampling from the robot's action space which might not always be possible in robotic problems. Our future work aims at relaxing these assumptions and address the planning problem under dynamics and environment uncertainty.

\section{ACKNOWLEDGMENT}

We would like to thank Xusheng Luo and Michael Zavlanos for their input and sharing their implementation of LTL motion planning [8] for experimental comparisons.

\section{REFERENCES}

[1] Amir Pnueli and Roni Rosner. On the synthesis of a reactive module. In Proceedings of the 16th ACM SIGPLAN-SIGACT symposium on Principles of programming languages, pages 179-190, 1989.

[2] Georgios E Fainekos, Hadas Kress-Gazit, and George J Pappas. Temporal logic motion planning for mobile robots. In Proceedings of the 2005 IEEE International Conference on Robotics and Automation, pages 2020-2025. IEEE, 2005.

[3] Hadas Kress-Gazit, Georgios E Fainekos, and George J Pappas. Temporal-logic-based reactive mission and motion planning. IEEE transactions on robotics, 25(6):1370-1381, 2009.
[4] Stephen L Smith, Jana Tumova, Calin Belta, and Daniela Rus. Optimal path planning for surveillance with temporal-logic constraints. The International Journal of Robotics Research, 30(14):1695-1708, 2011.

[5] Steven M. Lavalle. Rapidly-exploring random trees: A new tool for path planning. 1998.

[6] Sertac Karaman and Emilio Frazzoli. Sampling-based algorithms for optimal motion planning. The international journal of robotics research, 30(7):846-894, 2011.

[7] Amit Bhatia, Matthew R Maly, Lydia E Kavraki, and Moshe Y Vardi. Motion planning with complex goals. IEEE Robotics \& Automation Magazine, 18(3):55-64, 2011.

[8] Xusheng Luo, Yiannis Kantaros, and Michael M Zavlanos. An abstraction-free method for multi-robot temporal logic optimal control synthesis. arXiv preprint arXiv:1909.00526, 2019.

[9] Fernando S Barbosa, Lars Lindemann, Dimos V Dimarogonas, and Jana Tumova. Integrated motion planning and control under metric interval temporal logic specifications. In 2019 18th European Control Conference (ECC), pages 2042-2049. IEEE, 2019.

[10] Yiannis Kantaros and Michael M Zavlanos. Sampling-based optimal control synthesis for multirobot systems under global temporal tasks. IEEE Transactions on Automatic Control, 64(5):1916-1931, 2018.

[11] Amit Bhatia, Lydia E Kavraki, and Moshe Y Vardi. Sampling-based motion planning with temporal goals. In 2010 IEEE International Conference on Robotics and Automation, pages 2689-2696. IEEE, 2010.

[12] Ioan A. Sucan, Mark Moll, and Lydia E. Kavraki. The Open Motion Planning Library. IEEE Robotics \& Automation Magazine, 19(4):7282, December 2012.

[13] Russ Tedrake, Ian R Manchester, Mark Tobenkin, and John W Roberts. LQR-trees: Feedback motion planning via sums-of-squares verification. The International Journal of Robotics Research, 29(8):10381052, 2010.

[14] Sylvia L Herbert, Mo Chen, SooJean Han, Somil Bansal, Jaime F Fisac, and Claire J Tomlin. FaSTrack: A modular framework for fast and guaranteed safe motion planning. In 2017 IEEE 56th Annual Conference on Decision and Control (CDC), pages 1517-1522. IEEE, 2017.

[15] Anirudha Majumdar and Russ Tedrake. Funnel libraries for realtime robust feedback motion planning. The International Journal of Robotics Research, 36(8):947-982, 2017.

[16] Sumeet Singh, Anirudha Majumdar, Jean-Jacques Slotine, and Marco Pavone. Robust online motion planning via contraction theory and convex optimization. In 2017 IEEE International Conference on Robotics and Automation (ICRA), pages 5883-5890. IEEE, 2017.

[17] Pouria Tajvar, Anastasiia Varava, Danica Kragic, and Jana Tumova. Robust motion planning for non-holonomic robots with planar geometric constraints. In The International Symposium on Robotics Research October 6-10, 2019, Hanoi, Vietnam, 2019.

[18] Paul Gastin and Denis Oddoux. Fast LTL to Büchi automata translation. In International Conference on Computer Aided Verification, pages 53-65. Springer, 2001.

[19] Parosh Aziz Abdulla, Lisa Kaati, and Johanna Högberg. Bisimulation minimization of tree automata. In International Conference on Implementation and Application of Automata, pages 173-185. Springer, 2006.

[20] Maxim Likhachev, Geoffrey J Gordon, and Sebastian Thrun. Ara*: Anytime $\mathrm{a}^{*}$ with provable bounds on sub-optimality. In Advances in neural information processing systems, pages 767-774, 2004.

[21] Yasser Shoukry, Pierluigi Nuzzo, Ayca Balkan, Indranil Saha, Alberto L Sangiovanni-Vincentelli, Sanjit A Seshia, George J Pappas, and Paulo Tabuada. Linear temporal logic motion planning for teams of underactuated robots using satisfiability modulo convex programming. In 2017 IEEE 56th annual conference on decision and control (CDC), pages 1132-1137. IEEE, 2017.

[22] Nir Piterman, Amir Pnueli, and Yaniv Sa'ar. Synthesis of reactive (1) designs. In International Workshop on Verification, Model Checking, and Abstract Interpretation, pages 364-380. Springer, 2006.

[23] Orna Kupferman and Moshe Y Vardi. Model checking of safety properties. Formal Methods in System Design, 19(3):291-314, 2001. 